\title{
The Radiation Belt Storm Probes (RBSP) and Space Weather
}

\author{
R.L. Kessel • N.J. Fox • M. Weiss
}

Received: 13 March 2012 / Accepted: 27 November 2012 / Published online: 28 December 2012

(C) The Author(s) 2012. This article is published with open access at Springerlink.com

\begin{abstract}
Following the launch and commissioning of NASA's Radiation Belt Storm Probes (RBSP) in 2012, space weather data will be generated and broadcast from the spacecraft in near real-time. The RBSP mission targets one part of the space weather chain: the very high energy electrons and ions magnetically trapped within Earth's radiation belts. The understanding gained by RBSP will enable us to better predict the response of the radiation belts to solar storms in the future, and thereby protect space assets in the near-Earth environment. This chapter details the presently planned RBSP capabilities for generating and broadcasting near real-time space weather data, discusses the data products, the ground stations collecting the data, and the users/models that will incorporate the data into test-beds for radiation belt nowcasting and forecasting.
\end{abstract}

Keywords Space weather · Radiation Belt Storm Probes · RBSP · Geomagnetic storms

\section{Introduction}

Space weather is the state of the plasma, radiation, and magnetic environment in space driven by changes originating at the Sun and carried through interplanetary space by the solar wind. Space weather encompasses the solar environment where changes are initiated in the chromosphere or corona through to the near space environment above Earth (or any magnetized body). Space weather can cause large variations in Earth's upper atmosphere and ionosphere, and in Earth's radiation belts, and can prevent the reliable operation of technologies in space as well as on the ground. Our society is increasingly dependent on

R.L. Kessel

SMD, Heliophysics Division, NASA Headquarters, 300 E St. SW, Washington, DC 20546-0001, USA

e-mail: mona.kessel@nasa.gov

N.J. Fox $(\bowtie) \cdot$ M. Weiss

Applied Physics Laboratory, The Johns Hopkins University, Laurel, MD 20723, USA

e-mail: nicola.fox@jhuapl.edu 


\section{Locations of Operational Space Environment Hazards}

Single Event Effects (SEEs)
inner (proton) belt and higher $L$ shells with solar
particle event
quiet-times from galactic cosmic rays

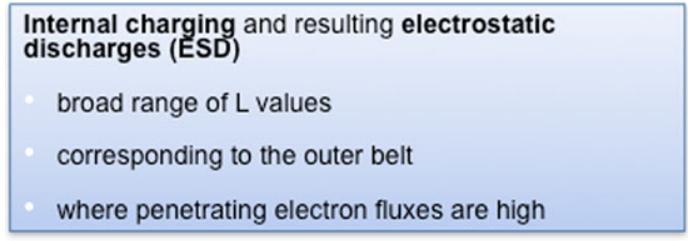

Surface charging and resulting ESD
spacecraft or surface potential elevated
$2000-0800$ local time in the plasma sheet
regions of intense field-aligned currents
observed, but not explained, at very low L

Total ionizing dose

electronics and solar panels degrade over time
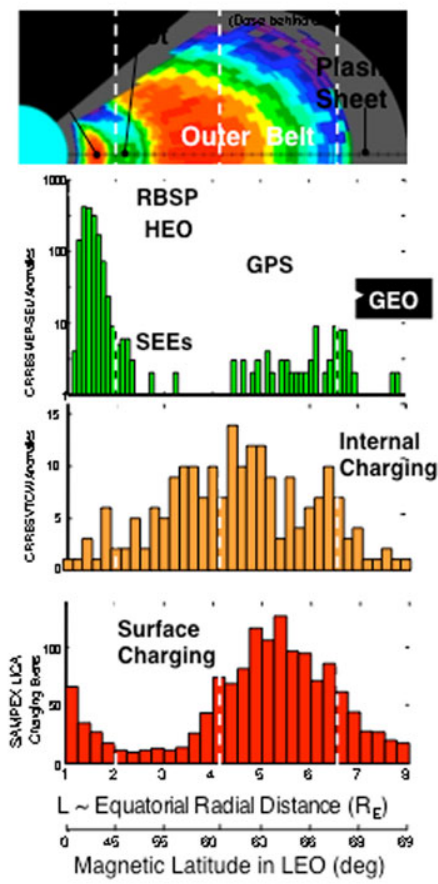

Fig. 1 Location of operational space environment hazards (courtesy of Aerospace Corporation)

these technologies, and their vulnerabilities to space weather need careful assessment, monitoring, and mitigation. Lanzerotti $(2001,2004)$ provide excellent reviews that describe the effects of space weather on past and current technologies.

The National Space Weather Program (NSWP) began in 1994 to help coordinate space weather activities and promote an increased awareness of space weather. An interagency working group within NSWP recognized early that modeling would play a key role in interpreting space weather data. The interagency working group recommended a modeling center that could transition research models to operations, as well as provide testing and validation. This recommendation led to the founding of the Community Coordinated Modeling Center (CCMC) (Robinson and Behnke 2001). Today the CCMC supports over 20 space weather models. NOAA's Space Weather Prediction Center (SWPC) is the U.S. official source for space weather forecasts, working with the CCMC and others to get the latest, most robust models enabling space weather prediction.

Recent interest at NOAA has focused on identifying space weather impacts to satellite infrastructure and defining services to mitigate those impacts. Figure 1 shows the locations of hazards within the operational space environment (courtesy of Aerospace, T.P. O'Brien). Single event effects predominate at low $L$ shell in the inner belt due to solar particle events, but can also be found from $L=4$ to 7 due to quiet time galactic cosmic rays. Internal charging and subsequent electrostatic discharges (ESD) can be found over a broad range of $L$ shells with a peak around $L=4.2$, likely where and when penetrating electron fluxes are high. Surface charging and ESD effects are largest in the outer belt with a peak at about 
$L=5.5$, probably due to intense field-aligned currents. At very low $L$, surface charging is also observed, but no explanation has yet been found.

NASA's Radiation Belt Storm Probes (RBSP) mission, launched on 30 August 2012, targets one part of the space weather chain: Earth's radiation belts of magnetically trapped, very high-energy electrons and ions. The unusual orbit of the RBSP spacecraft will provide great insight into many regions of the radiation belts. The highly elliptical orbit of $\operatorname{RBSP}\left(600 \mathrm{~km}\right.$ altitude $\times 5.8 R_{E}$ geocentric; and $10^{\circ}$ inclination) is non-traditional-most communication and monitoring satellites operate at fixed radial distance, for example at or near geosynchronous orbit at $\sim 6.7 R_{E}$ geocentric. For $3-\mathrm{D}$ specification models, the altitude-varying profiles returned by RBSP will provide greater sampling of Earth's radiation environment. For RBSP this orbit is occupied by two identical spacecraft that lap each other every $\sim 2.5$ months. Detailed information about the RBSP science, mission and spacecraft design is described elsewhere in this special issue (Mauk et al. 2012; Stratton et al. 2012, and Kirby et al. 2012).

In addition to scientific data (provided at a mean rate of about 100 kilo bits per secondkbps), each RBSP spacecraft will provide a continuous $1 \mathrm{kbps}$ of space weather broadcast data in support of near real time space weather modeling, forecast and prediction efforts. The real-time data from RBSP also will be available to monitor and analyze current environmental conditions, forecast natural environmental changes and support anomaly resolution. RBSP real-time data will be input into the DREAM model (Sect. 4.4), the output of which will be made available through NOAA (Sect. 4.3). This will provide a simple and very quick visual for displaying the current state of the inner and outer radiation belts and the spacecraft charging environment.

Following the commissioning of RBSP in 2012, the RBSP instruments will generate real-time space weather observations to be broadcast by both RBSP spacecraft. This chapter describes presently planned RBSP capabilities for generating and broadcasting the realtime space weather data (Sect. 2). These data will be collected by ground station partners (Sect. 4.1), and gathered and processed at the Johns Hopkins University Applied Physics Laboratory (Sect. 3). The RBSP Science Gateway website (http://rbspgway.jhuapl.edu) will host and continuously update the Space Weather products that will be used to feed into models and assess space weather conditions. Modeling capabilities are discussed in Sect. 4, and concluding remarks are provided in the final section.

\section{Generation and Broadcast of Space Weather Data}

Each spacecraft will broadcast space weather data in real-time through the primary spacecraft radio frequency (RF) science downlink system, whenever it is not engaged in a primary mission-related ground contact. The data will be received by users who maintain and fund their own ground station antennas. This scenario is limited by the availability of space weather ground stations and antenna coverage. The real time coverage will be reduced by an average of 2.5 hours for each spacecraft per day due to primary mission contacts, or about $10 \%$ of the time. Often when one of the spacecraft is broadcasting the primary science data, and therefore not broadcasting space weather data, the other spacecraft will still be broadcasting space weather data because many of the contacts with each spacecraft do not overlap in time.

Each of the RBSP payload instruments will participate in the real-time space weather broadcast. The data will include particle intensities at a variety of energies, as well as magnetic and electric field data. In addition to the real-time products, it is a goal for the project 
Fig. 2 Statistical distribution with uniform time sampling of RBSP downlink acquisitions plotted as a function of the angle between the RBSP Spin axis (the axis along which the onboard communications antennas are aligned-Fig. 3; called the Mast Angle) and the distance between the spacecraft and the ground station. The antenna pattern for the two antennas on each spacecraft is such that the transmission power is very low within the "null region". It is conservatively assumed that communications may not take place within that region

Fig. 3 Schematic of the RBSP radio frequency communications antenna coverage. The sketched patterns are rotationally symmetric with respect to rotations about the horizontal (spacecraft " $z$ ") axis
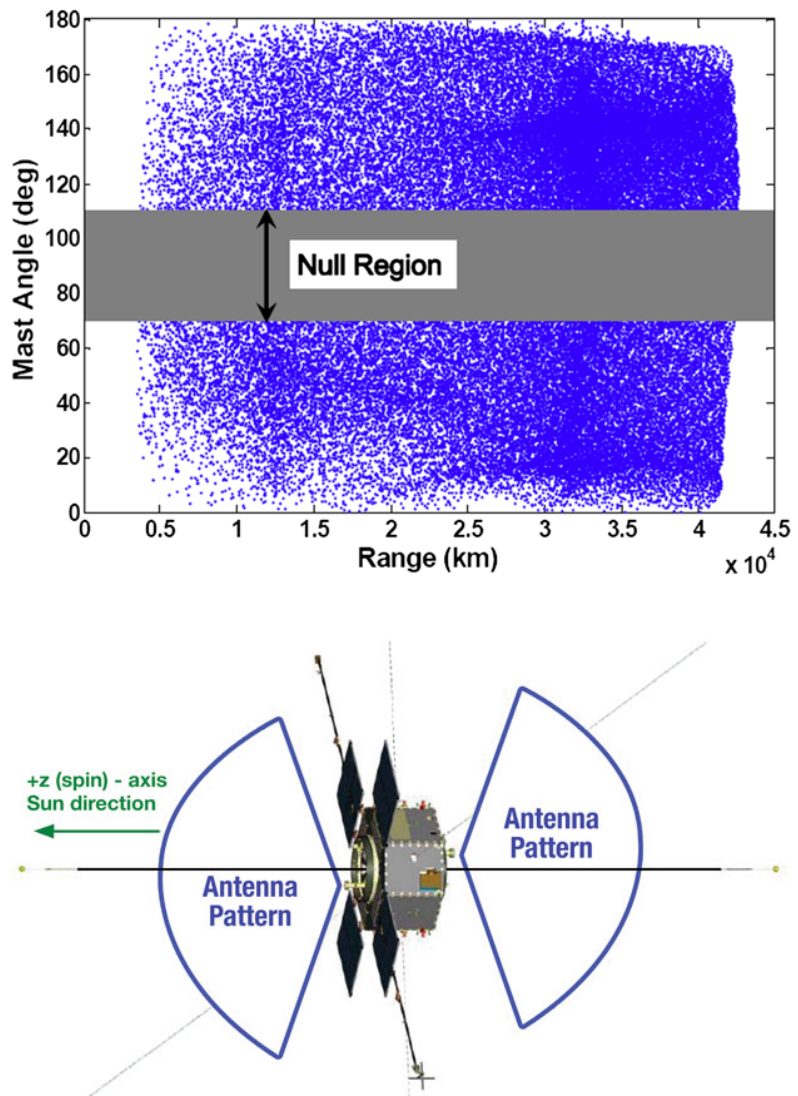

to create "quick look" products to be produced by each of the individual instrument Science Operations Centers (SOC). These products will essentially "fill in the gaps" caused by times when the broadcast data cannot be received and also provide a more complete data set for use in diagnosing anomalies in low (LEO) and mid (MEO) Earth orbit.

The RBSP mission spacecraft will communicate with the ground via S-Band using $8 \mathrm{~W}$ Solid State Power Amplifier (SSPA) transmitters. The spacecraft pointing geometry, orbit, and spin stabilization determine communication system requirements. Earth location, as viewed from the spacecraft, covers a very broad angle space (mast angle) as shown in the RBSP communications antenna angle coverage plot in Fig. 2. Contact geometry necessitates onboard antennas that have broad angular coverage and thus relatively low gain.

The coverage is maximized within practical limits using two low gain antennas. The two RF antennas' boresights are aligned with the spacecraft spin- and anti-spin-axes, providing coverage from each boresight to $70^{\circ}$ (Fig. 3). Despite maximizing the antenna coverage, there is still a $40^{\circ}$ null band, depicted in Fig. 2 as the gray patch in the antenna angle coverage plot. Both antennas are active at all times, as there is no active switching between antennas, but only one will have a line-of-sight to the Earth at any given time (Srinivasan et al. 2009). The top antenna and bottom antenna generate circularly polarized emissions with one spacecraft, RBSP A, using Right Hand Circular Polarization and for the other spacecraft, RBSP B, using Left Hand Circular Polarization. The spacecraft and antenna patterns are illustrated in Fig. 3. 
Fig. 4 Regions of the RBSP Orbits where communications downlinks are robust, variable, and impossible. The communications configuration changes over time because of the roughly $220^{\circ}$ per year of clockwise (from the north) local time precession of the line of apogee

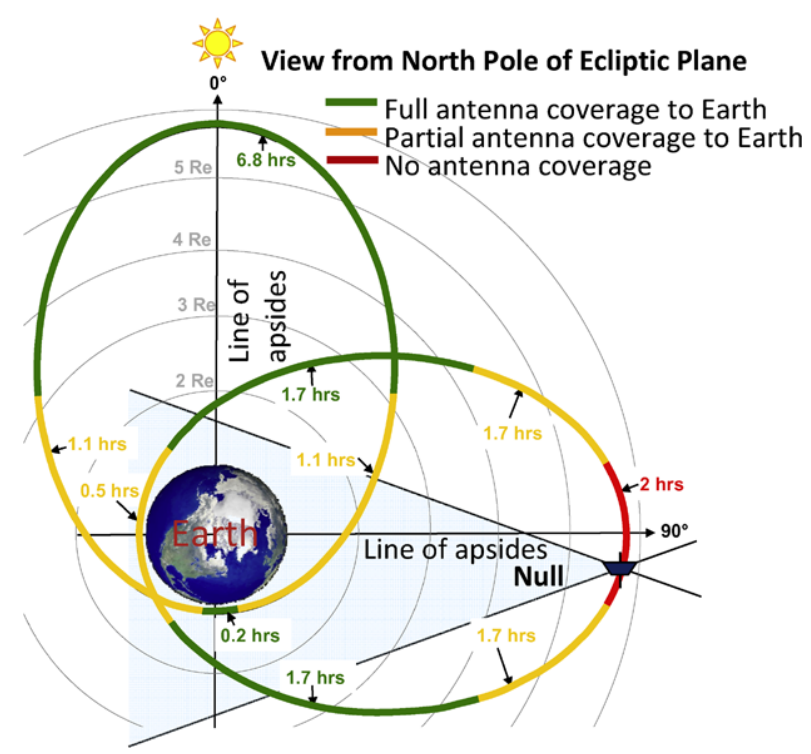

The possibility of users on Earth receiving the space weather broadcasts depends on an orbit geometry that varies with mission epoch as illustrated in Fig. 4-a view of the orbit changes as seen from a vantage pointing looking down on the Earth from above the North Pole. Although antenna coverage is large, there are times when the antenna patterns are not aligned with the Earth. The large eccentricity of the orbit causes longer periods of time when antennas are not in view during certain times of the year because the relative angle between the spacecraft and Earth changes slowly over long periods of time. Spacecraft orbits are at a low inclination $\left(10^{\circ}\right)$ and the orbit harmonics cause the apogee and perigee to drift between northern and southern hemispheres. Stations near the equator will have the best year-around coverage while high latitude stations may have some limits in coverage over portions of the orbit for parts of the year.

By employing multiple stations at diverse longitudes, we can maximize potential spacecraft contact duration as a function of the number of ground stations. Figure 5 shows the coverage for $1,2,3$ and 4 ground stations with maximum angular separation-i.e. 2 separated by $180^{\circ} ; 3$ by $120^{\circ}$; and 4 by $90^{\circ}$. The peaks and valleys in potential contact periods are primarily due to orbit epoch effects on spacecraft antenna coverage. Normal spacecraft contacts (i.e. downlinking of science data and uplinking commands etc.) would reduce available time for space weather by an average of 2.5 hours per spacecraft per day $(\sim 10 \%$ per spacecraft).

\section{RBSP Space Weather Data Products and Services}

The space weather data products were chosen to provide a quick look at the state of the radiation belts. The data subset includes particle intensities at a variety of energies, and magnetic and electric field data, both quasi-time-stationary parameters and wave parameters. Current data products can be found in Table 1 and at http://rbspgway.jhuapl.edu. The exact energy and frequency ranges are subject to refinement as the instrument teams plans continue to mature. 


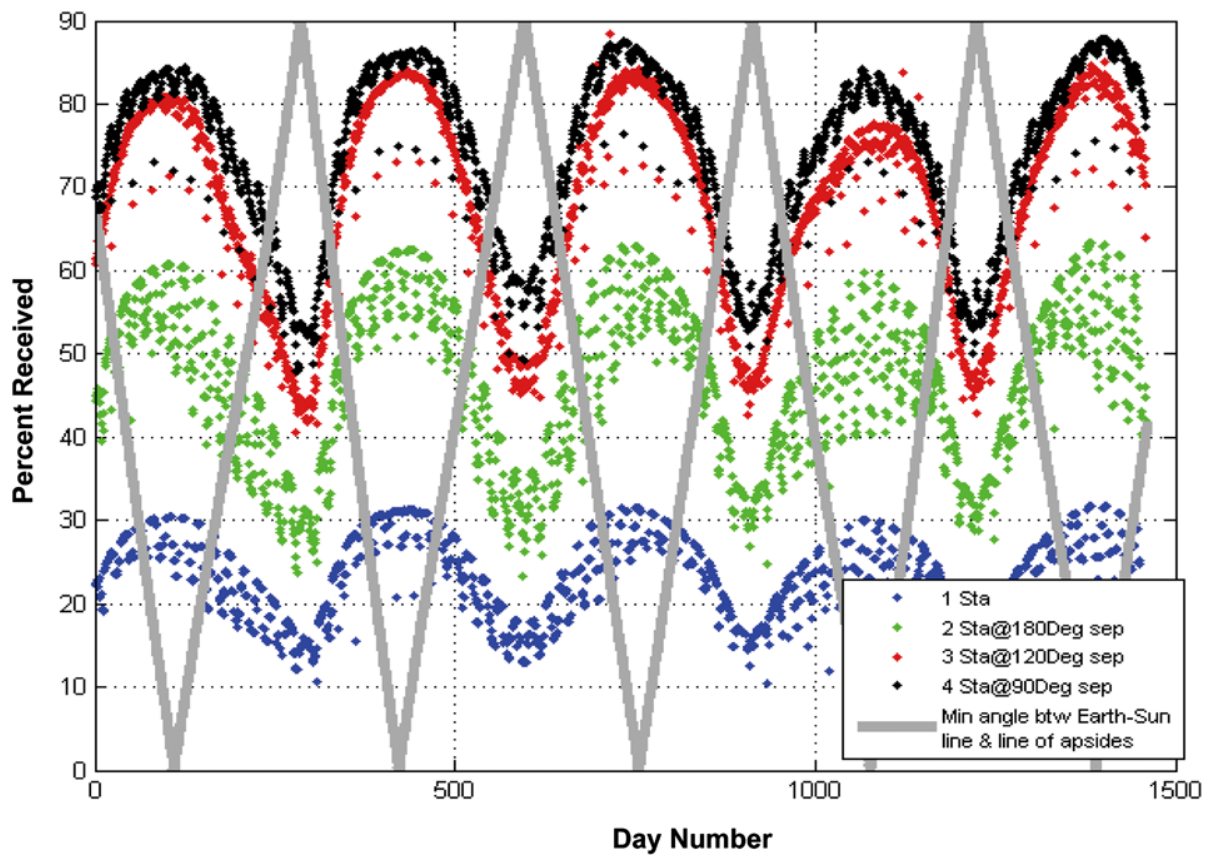

Fig. 5 Average contact time per day (\%) of the RBSP spacecraft to 1 or more ground stations distributed around the world plus the minimum angle between Earth-Sun line and line of Apsides (deg). Assumptions: (1) Visibility averaged over 3 day increments. (2) Two $70^{\circ}$ half angle antennas. (3) Generic ground stations assumed at $35^{\circ} \mathrm{N}$ latitude

The energies within the space weather data by the particle detectors span the ranges expected for the two belts, $25 \mathrm{eV}$ to $>400 \mathrm{MeV}$ for protons, $25 \mathrm{eV}-10 \mathrm{MeV}$ for electrons. ECT/HOPE (Funsten et al. 2012-this issue) provides the lower range of both the inner belt ions and outer belt electrons, and the ion composition for the lower energy ions. ECT/MagEIS (Blake et al. 2012 - this issue) provides the mid and upper range of outer belt electrons. ECT/REPT (Baker et al. 2012 - this issue) provides the upper range of inner belt protons and high range of outer belt electrons, while PSBR/RPS (Mazur et al. 2012this issue) cover the extreme upper range of inner belt protons. RBSPICE (Lanzerotti et al. 2012-this issue) provides the mid-energy protons intensities.

The EFW instrument (Wygant et al. 2012 - this issue) provides the large-scale electric field, and the magnetometer on EMFISIS (Kletzing et al. 2012-this issue) provides the large-scale magnetic field. The Waves instrument portion of EMFISIS on RBSP will provide three values of the high frequency electric and magnetic field observations every 12 seconds for the RBSP space weather data set. The three bands chosen, based on present understanding about which waves propagating within the regions of the radiation belts have the most impact, are defined as follows:

- fce to 0.5 fce (lower band chorus)

- 0.5 fce to 0.7 fce (upper band chorus)

- $10 \mathrm{~Hz}$ to fce/(1837) ${ }^{1 / 2}$ (magnetosonic waves)

Here fce is the so-called electron cyclotron frequency and 1837 is the proton to electron mass ratio. Each band, then, can provide the intensity of the chorus band or magnetosonic waves 
Table 1 Space weather broadcast data products

\begin{tabular}{|c|c|c|c|}
\hline Instrument & Measurement & Energy & Cadence \\
\hline EMFISIS/MAG & $\begin{array}{l}\text { Vector Magnetic } \\
\text { Field }\end{array}$ & N/A & 1 vector/12 seconds \\
\hline EMFISIS/Waves & VLF Wave Power & N/A & $\begin{array}{l}\text { E-field spectral density: } \\
3 \text { frequencies every } \\
12 \mathrm{~s} \text {. B-field spectral } \\
\text { density: } 3 \text { frequencies } \\
\text { every } 12 \mathrm{~s}\end{array}$ \\
\hline \multirow[t]{2}{*}{ EFW } & $\begin{array}{l}\text { Vector Electric } \\
\text { Field }\end{array}$ & N/A & 1 vector/spin \\
\hline & Spacecraft Potential & N/A & Once/spin \\
\hline \multirow[t]{4}{*}{ ECT/HOPE } & Electrons & $\begin{array}{l}24.54 \mathrm{eV}, 281 \mathrm{eV}, 10.9 \mathrm{keV} \text {, } \\
42.9 \mathrm{keV}\end{array}$ & Once/24 seconds ${ }^{*}$ \\
\hline & Protons & $\begin{array}{l}24.54 \mathrm{eV}, 281 \mathrm{eV}, 10.9 \mathrm{keV} \\
42.9 \mathrm{keV}\end{array}$ & \\
\hline & Oxygen Ions & $\begin{array}{l}24.54 \mathrm{eV}, 281 \mathrm{eV}, 10.9 \mathrm{keV}, \\
42.9 \mathrm{keV}\end{array}$ & \\
\hline & Helium Ions & $\begin{array}{l}24.54 \mathrm{eV}, 281 \mathrm{eV}, 10.9 \mathrm{keV} \text {, } \\
42.9 \mathrm{keV}\end{array}$ & \\
\hline \multirow[t]{2}{*}{ ECT/MagEIS } & Energetic Electrons & $\begin{array}{l}30 \mathrm{keV}, 60 \mathrm{keV}, 100 \mathrm{keV}, \\
300 \mathrm{keV}, 600 \mathrm{keV}, 1 \mathrm{MeV}, \\
2 \mathrm{MeV}\end{array}$ & Once/spin \\
\hline & Energetic Protons & $1 \mathrm{MeV}$ & \\
\hline \multirow[t]{2}{*}{ ECT/REPT } & $\begin{array}{l}\text { Very Energetic } \\
\text { Electrons }\end{array}$ & $2 \mathrm{MeV}, 5 \mathrm{MeV}, 10 \mathrm{MeV}$ & Once/spin \\
\hline & Energetic Protons & $\begin{array}{l}>20 \mathrm{MeV},>50 \mathrm{MeV}, \\
>70 \mathrm{MeV}\end{array}$ & \\
\hline RBSPICE & Energetic Protons & $\begin{array}{l}50 \mathrm{keV}, 100 \mathrm{keV}, 150 \mathrm{keV} \\
300 \mathrm{keV}, 1 \mathrm{MeV}, 10 \mathrm{MeV}\end{array}$ & Once/spin \\
\hline PSBR/RPS & $\begin{array}{l}\text { Energetic Protons } \\
\text { Dosimeter Data }\end{array}$ & $\begin{array}{l}>50 \mathrm{MeV},>400 \mathrm{MeV} \\
\text { Linear \& Log Volts }\end{array}$ & Once/1 seconds \\
\hline
\end{tabular}

\footnotetext{
*Ions and electrons are sampled alternately every other spin
}

as a function of time (hence, position, as well). By themselves, these observations form the basis of a statistical sampling of the intensity of these phenomena as a function of position. When compared with a model of the occurrence of these phenomena, the parameters allow for instantaneous determination of wave levels relative to the model.

The display of the space weather data is still under discussion, but will likely include products such as: (i) 3 -dimensional $L$-shell-position ( $y$-axis) versus orbit or time ( $x$-axis) versus selected parameters such as particle intensity (represented in the 3rd dimension with a color scale), with the latest data plotted on the right hand side (an example using CRRES data is shown in Fig. 6), (ii) selected parameters such as particle intensity over-layed on the orbit path with the value of that parameter indicated with variable colors or line thicknesses, (iii) line plots of such selected parameters as magnetic field vector and electric field vector, spacecraft charging values, as well as individual team summary plots. The plots will be updated approximately every 12 minutes when space weather data is available. The RBSP space weather telemetry mode is a very low rate, highly compressed data stream broadcast by the spacecraft 24 hours per day. Because of the large compression factors used, the images 


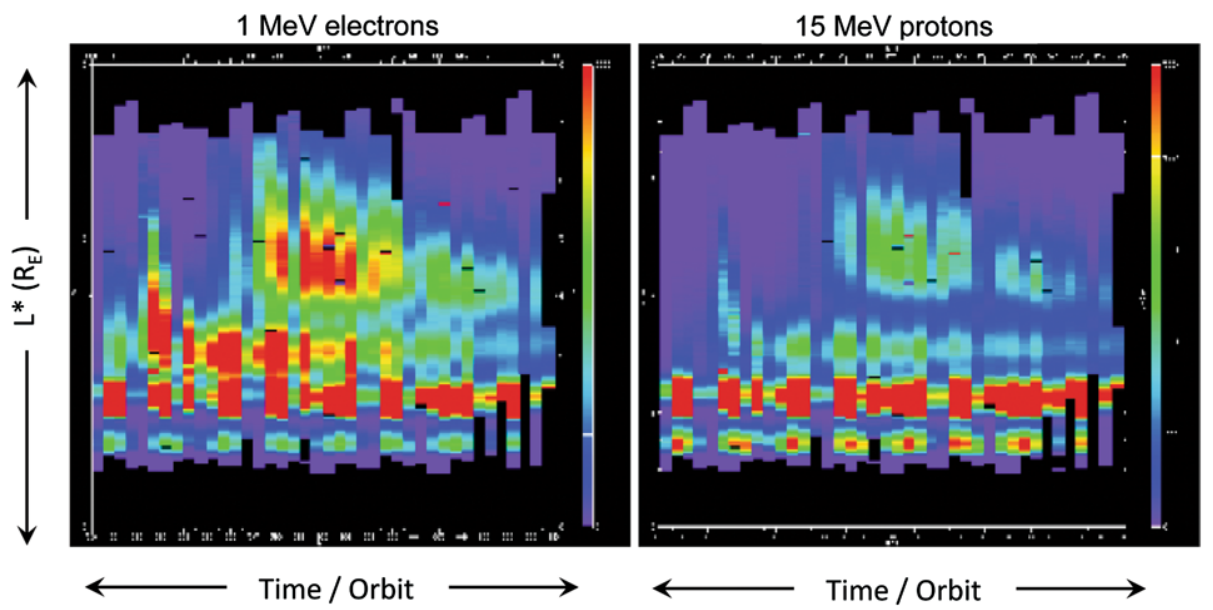

Fig. 6 Images created from CRRES mission showing $L^{*}$ ( $y$-axis; in $\left.R_{E}\right)$ versus time or orbit ( $x$-axis; each vertical stripe will be $\frac{1}{2}$ of a 9 hour orbit for RBSP; $x$ axis) versus intensity (color scale) of $1 \mathrm{MeV}$ electrons (left) and $15 \mathrm{MeV}$ protons (right). When RBSP launches, the RBSP instruments will be generating and broadcasting real-time space weather data. The RBSP Space Weather images will be created from that data and available at http://rbspgway.jhuapl.edu/weather_currentdata

like that shown in Fig. 6 are of much lower quality and fidelity than those that will be generated by the teams with the actual science data. The raw space weather data will be archived at APL.

The space weather processing uses legacy APL MOC (Mission Operations Center) software developed for the STEREO mission and the LRO/Chandrayaan-1 Mini-RF project. The space weather processing software flow is notionally depicted in Fig. 7. The MOC software will routinely run and automatically ping on the external ground stations to see if new data is available. When available, it will retrieve the data from the external ground stations, strip out the headers put into the Space Weather packets by the external ground stations, extract the variable length packets from the data, clean and merge the space weather Level 0 data and store the raw data in a telemetry archive.

The space weather processing system will periodically ping on the MOC telemetry archive and when new data is available; it will retrieve that data from the archive, decommutate the data and then apply calibration algorithms to generate space weather data products for all of the RBSP instruments. The current space weather data products will be publicly available at http://rbspgway.jhuapl.edu/weather_currentdata. The space weather products will be archived; the most recent 15 days will be publicly available on the RBSP Science Gateway at http://rbspgway.jhuapl.edu/weather_archive.

\section{Partners and Customers}

The RBSP mission will provide space weather parameters to the user community for integration into nowcast and forecast models. In order to collect these data, a network of ground stations needs to be identified. 


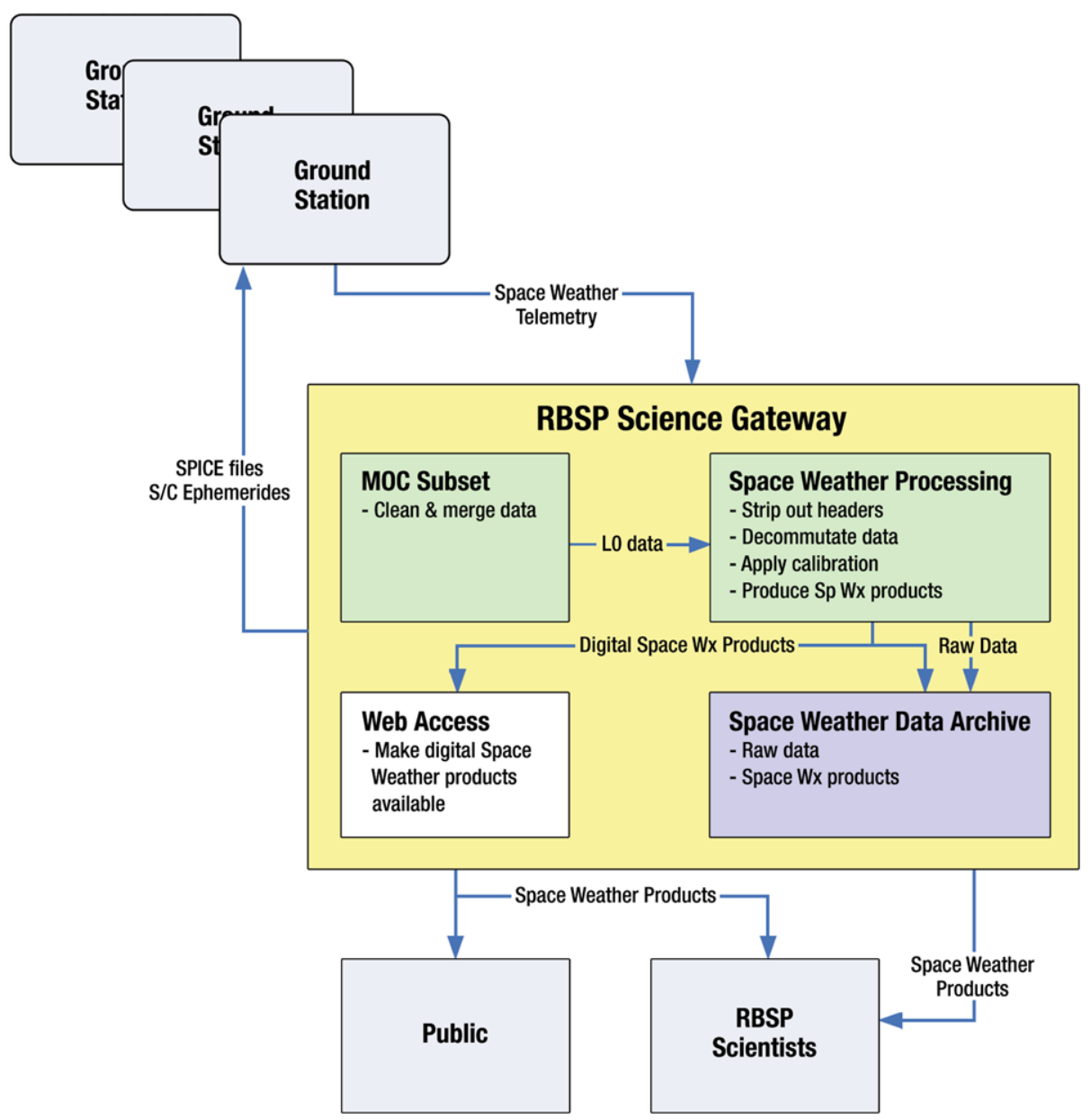

Fig. 7 RBSP space weather data acquisition and processing plan. The ground stations are contributed assets distributed around the world, and the rest of the processing occurs at APL and will be available at http://rbspgway.jhuapl.edu/weather_currentdata

\subsection{Ground Stations}

Ideally, ground stations should be distributed around the globe at longitudinal separations of about $120^{\circ}$ for an optimized 3 -station configuration. The telemetry link is subject to orbit geometry, season, and location of each ground station. It is estimated that with an ideal ground network, the link could be operational for about $65 \%$ of the time (assuming 3 longitudinally spaced stations). The normal spacecraft contacts will reduce available time for space weather by an average of 2.5 hours per spacecraft per day.

Ground stations are supplied, operated, and funded by interested parties external to the RBSP Program and Project. Currently, two international ground station partners have been identified and agreements are in place:

- Korea Astronomy and Space Science Institute

- Institute of Atmospheric Physics, Czech Republic 
Discussions are underway with other possible international partners including Brazil, Japan, Argentina, Australia, South Africa, and India.

APL regularly provides the participating ground stations with the spacecraft ephemerides and will routinely retrieve the Space Weather data from those ground stations using either socket connections or using ftp/sftp protocols. The APL processing flow for the space weather processing is described in Sect. 3 above.

The RBSP Space Weather ICD contains specific downlink and telemetry formats and is available to partners on request.

\section{2 $\mathrm{CCMC}$}

The Community Coordinated Modeling Center (CCMC) is a US inter-agency activity, located at GSFC, aimed at research in support of the generation of advanced space weather models (http://ccmc.gsfc.nasa.gov/). The first function of the CCMC is to provide a mechanism by which research models can be validated, tested, and improved for eventual use in space weather forecasting. Examples include NASA's Vision for Space Exploration Models. These models, which have completed their development and which have passed metricsbased evaluations and science-based validations, are being prepared for space weather applications. In this function, CCMC acts as an unbiased evaluator, which bridges the gap between space science research and space weather applications.

As a second equally important function, the CCMC provides to space science researchers the use of space science models, even if those researchers are not model owners themselves. This service to the research community is implemented through the execution of model "runs-on-request" for specific events of interest to space science researchers at no cost to the requestor. Model output is made available to the science customer by means of tailored analysis tools, and by means of data dissemination in standard formats. Through this activity and the concurrent development of advanced visualization tools, CCMC provides unprecedented access to a large number of state-of-the-art research models to the general science community. The continuously expanding model set includes models in all scientific domains from the Solar Corona to the Earth's upper atmosphere. Data received from RBSP will be available for scientific comparisons with model calculations, and as inputs to model calculations performed following requests from the scientific community.

Models tested and evaluated at CCMC are being used at NASA's Space Weather Research Center (SWRC) for providing critical space weather notification for NASA's robotic missions. The SWRC provides a broad range of tools, products, and services including routine experimental research forecasts, notifications, space weather analysis, and spacecraft anomaly resolution support. The SWRC also makes advanced model results and data streams available for public education and information purposes. The SWRC will be receiving RBSP space weather data for situational awareness, model validation, and for ingestion into specification and forecasting models.

\subsection{NOAA}

NOAA's Space Weather Prediction Center (http://swpc.noaa.gov) is the United States government official source for space weather forecasts. SWPC provides real-time monitoring and forecasting of solar and geophysical events that impact satellites, power grids, communications, navigation, and many other technological systems. There is a range of online data products and services including: Alerts and Forecasts, Models, Indices, and real-time or near-real time instrument measurements. RBSP data will augment their current capabilities 
of understanding the space radiation environment, primarily through display of the DREAM model (discussed below) into which near-real time and retrospective MagEIS data has been ingested. This would provide a simple visual for the current level of charged particles in the radiation belts, and hence current internal charging conditions. A new service is under development within the National Geophysical Data center (NGDC) that will combine nearreal time and retrospective data for post satellite anomaly analysis. A web page will provide interactive plots of data and models for GEO and LEO orbits for determining whether an anomaly is likely related to surface charging, internal charging, single event effects or total ionizing dose. This will help SWPC and NGDC to deliver space weather products and services that meet the evolving needs of its government and industry stakeholders.

\subsection{DREAM}

A particularly relevant model for analysis of space weather data from the RBSP mission is the Dynamic Radiation Environment Assimilation Model (DREAM). It was developed to provide accurate, global specification of the Earth's radiation belts and to better understand the physical processes that control radiation belt structure and dynamics (Reeves et al. 2012). DREAM will be used in the RBSP science analysis in two major roles: (a) as a global context for understanding the local 2-satellite measurements and (b) as a testbed for real-time space weather forecasting for the radiation belts. As the name implies, DREAM uses a powerful data assimilation technique (specifically ensemble Kalman Filtering) to calculate a global specification of the radiation belt environment that optimizes the match between model and observations. Unlike traditional models that use "inputs" or "boundary conditions", data assimilation considers uncertainties in both model and observation, it includes observations as part of the internal state of the system, and observations (through a covariance matrix) that affect extended regions around the location of observations.

DREAM uses a Fokker-Planck diffusion formulation (Reeves et al. 2012; see also Ukhorskiy and Sitnov 2012-this issue) as the physics engine that advances the forecast in time. At the time of this writing, data assimilation has only been implemented in the 1D (radial diffusion) version but a 3D-version with radial, energy, and pitch angle diffusion is undergoing testing. The radial diffusion calculation solves for phase space density (PSD) as a function of three magnetic invariants: $\mu, K$, and $L^{*}$ (invariants of gyration, bounce and drift; again see Ukhorskiy and Sitnov 2012 - this issues). DREAM first preprocesses data from intensity (as a function of energy, $E$, and pitch angle, $\alpha$ ) to PSD as a function of $\mu, K, L^{*}$. [As part of the standard RBSP data processing, $\mu, K$, and $L^{*}$ will be calculated along the satellite orbits using a variety of different magnetic field models and values, and will be made available for analysis.] Data assimilation runs are done at each time step for each $\mu, K$ pair (typically 324 independent assimilations). In an asymmetric (and time varying) magnetic field, each point in space and time has a unique transformation from physical to magnetic coordinates. The same is true for the reverse transformation that takes the DREAM assimilation and converts back to intensity (vs. $E$ and $\alpha$ ). Figure 8 (from Reeves et al. 2012) shows an example of the incorporation of the Phase Space Density measurements shown on the left to create a prediction (on the right) for the Phase Space Densities throughout the radiation belt regions. RBSP will give the predictions a lot more fidelity by filling in a lot of the gaps between and inside of the present measurement positions.

The result is a model that gives the space weather forecast for the radiation beltsintensity, flux and fluence, or dose-at any point in the radiation belts based on a very limited set of observations. For space weather forecasting the model can be relatively simple with few (or no) free parameters-for example 1D radial diffusion with $D_{L L}(\mathrm{Kp})$, such 

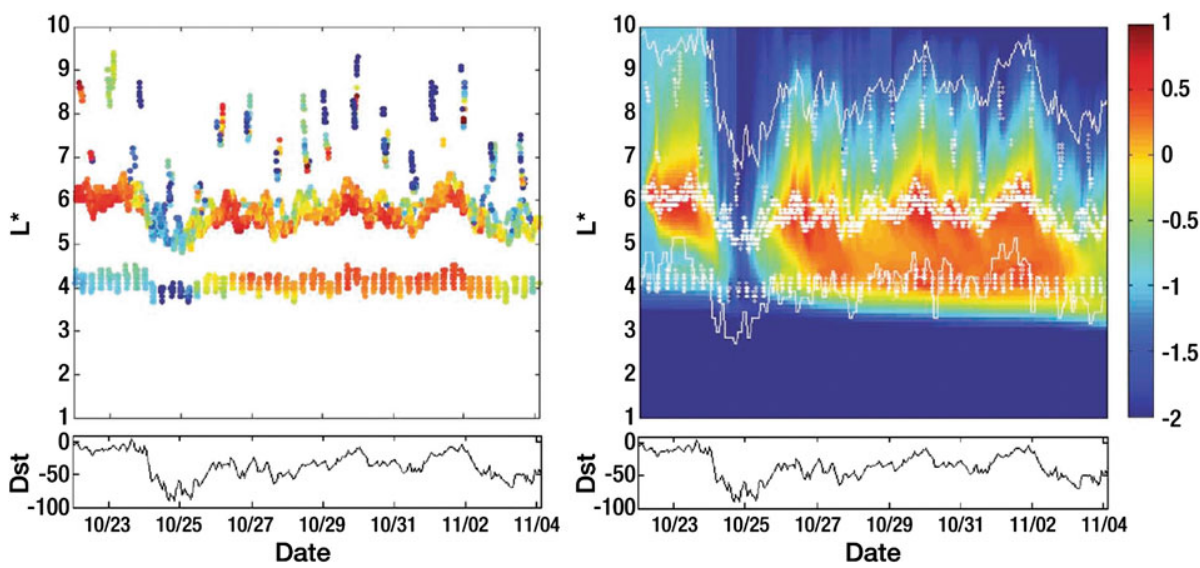

Fig. 8 Example of the capability of the DREAM model (Reeves et al. 2012) to assimilate sparse spacecraft measurements (left) together with empirical particle transport and energization algorithms to generate predictions of the overall state of Earth's electron radiation belt. The plots show the magnetospheric parameter $L^{*}$ ( $y$-axis) versus time ( $x$-axis) versus particle phase space density (color scale), calculated from the particle intensity and the particle momentum. Figures from Reeves et al. (2012)

as is now available with the DREAM model. For detailed scientific analysis more complex models with 3D diffusion and many free parameters will likely be needed with, for example, spatial and temporal distributions of wave power, frequency, and wave normal angle for a variety of wave modes). One of the goals of the RBSP project is to evolve space weather products from the current state of nowcasting with simple models to more sophisticated products that use more complex physics models (balancing accuracy and complexity) and that provide forecasts days or more into the future.

\section{Concluding Remarks}

The two spacecraft that comprise NASA's LWS RBSP mission will continuously broadcast space weather data, except during prime science download and maneuvers. These data were selected to monitor the state of the radiation belts and will be incorporated into models such as DREAM that could lead to better space weather forecasts.

Currently two international partners have agreed to download this data and make it available for space weather data products. With only two ground stations, portions of the data stream will be lost, but NASA is actively pursuing other ground station partners to fill in the gaps.

RBSP has been designed to operate throughout the worst conditions expected in the hazardous radiation belt environment (Stratton et al. 2012 and Kirby et al. 2012-this issue). By design, the mission will make observations over the full range of particle energy levels and frequencies needed to decipher the mysteries described elsewhere in this volume (Mauk et al. 2012 - this issue). RBSP is poised to significantly enhance our understanding of radiation belt dynamics with changing solar wind conditions. RBSP will enable the prediction of extreme and dynamic space conditions, and will provide the understanding needed to design satellites to survive in space for future missions.

Acknowledgements The authors gratefully acknowledge contributions from M. Hesse, G. Reeves, W. Kurth, J. Green, and T.P. O’Brien. 
Open Access This article is distributed under the terms of the Creative Commons Attribution License which permits any use, distribution, and reproduction in any medium, provided the original author(s) and the source are credited.

\section{References}

D.N. Baker et al., Space Sci. Rev. (2012, this issue). doi:10.1007/s11214-012-9950-9

J.B. Blake et al., Space Sci. Rev. (2012, this issue)

H. Funsten et al., Space Sci. Rev. (2012, this issue)

K. Kirby et al., Space Sci. Rev. (2012, this issue). doi:10.1007/s11214-012-9949-2

C. Kletzing et al., Space Sci. Rev. (2012, this issue)

L.J. Lanzerotti, Solar and solar radio effects on technologies, in Solar and Space Weather Radiophysics, ed. by P. Gary, P. Keller (Kluwer Academic, Dordrecht, 2004), p. 1

L.J. Lanzerotti, Space weather effects on technologies, in Space Weather, ed. by Song, Singer, Siscoe. Geophysical Monograph, vol. 125 (American Geophysical Union, Washington, 2001), p. 11

L.J. Lanzerotti et al., Space Sci. Rev. (2012, this issue)

B.H. Mauk, N.J. Fox, S.G. Kanekal, R.L. Kessel, D.G. Sibeck, A. Ukhorskiy, Science objectives and rationale for the Radiation Belt Storm Probes mission. Space Sci. Rev. (2012). doi:10.1007/s11214-012-9908-y

J. Mazur, L. Friesen, A. Lin, D. Mabry, N. Katz, Y. Dotan, J. George, J.B. Blake, M. Looper, M. Redding et al., The Relativistic Proton Spectrometer (RPS) for the Radiation Belt Storm Probes mission. Space Sci. Rev. (2012). doi:10.1007/s11214-012-9926-9

G.D. Reeves, Y. Chen, G.S. Cunningham, R.W.H. Friedel, M.G. Henderson, V.K. Jordanova, J. Koller, S.K. Morley, M.F. Thomsen, S. Zaharia, The Dynamic Radiation Environment Assimilation Model: DREAM. Space Weather (2012). doi:10.1029/2011SW000729

R.M. Robinson, R.A. Behnke, The U.S. National Space Weather Program: a retrospective, in Space Weather, ed. by P. Song, H. Singer, G. Siscoe. Geophysical Monograph, vol. 125 (American Geophysical Union, Washington, 2001), p. 1

D. Srinivasan, B. Wallis, B. Baker, D. Artis, RF communications subsystem for the Radiation Belt Storm Probes mission. Acta Astron. 65(11-12) (2009)

J. Stratton et al., Space Sci. Rev. (2012, this issue). doi:10.1007/s11214-012-9933-x

A. Ukhorskiy, M. Sitnov, Space Sci. Rev. (2012, this issue)

J. Wygant et al., Space Sci. Rev. (2012, this issue) 